\title{
Numerical Investigation of Wall Shear Stress under Single-Phase and Two-Phase Flow in the Electrical Submersible Pump
}

\author{
ABDULQADER HASAN \\ Department of Mechanical \\ Engineering \\ Abu Dhabi University \\ Abu Dhabi, P. O. Box 59911 \\ UNITED ARAB EMIRATES
}

\author{
MOHAMED S. GADALA \\ Department of Mechanical \\ Engineering \\ Abu Dhabi University \\ Abu Dhabi, P. O. Box 59911 \\ UNITED ARAB EMIRATES
}

\author{
SALMAN SHAHID \\ Department of Mechanical \\ Engineering \\ Abu Dhabi University \\ Abu Dhabi, P. O. Box 59911 \\ UNITED ARAB EMIRATES
}

\author{
MOHD SHIRAZ ARIS \\ TNB Research Sdn. Bhd. \\ Kawasan Institusi Penyelidikan \\ 43000 Kajang \\ MALYSIA
}

\author{
SHARUL SHAM DOL \\ Department of Mechanical \\ Engineering \\ Abu Dhabi University \\ Abu Dhabi, P. O. Box 59911 \\ UNITED ARAB EMIRATES
}

Abstract: - The effects of the wall shear stress on an Electrical Submersible Pump (ESP) was investigated in this paper. A CFD model in ANSYS Fluent was proposed to simulate actual single-phase and two-phase flow. The bottom hole pressure was minimized by utilizing the artificial lift methods. The flowing fluids in pumps and pipes causes shear stress on surface interacting. In multiphase flow application pump damages on head degradation as well as shear stress affects. The $K-\omega$ turbulence model and the multiphase Mixture approach with the sliding technique used to solve the Navier-Stokes equation. To study the effects of gas-liquid (air-water) flow on the ESP and the pump handle ability, the rotation speeds were varied while the other parameters were kept constant. The rotation speeds simulated were at 500, 900, 1500, 2000 and $2500 \mathrm{rpm}$ meanwhile the water flow rate and gas flow rate were kept constant with $20 \mathrm{~L} / \mathrm{min}$ and $1 \%$ fraction, respectively. The results obtained show that as the rotation speeds were increased, the less concentration of the bubbles were observed, moreover the wall shear stress (WSS) increases. Although, the wall shear stress in both single-phase and two-phase flow were tend to increase as the blades length increased, however for the single-phase flow the WSS was found higher in all the simulated rotational speeds.

Keywords: Cavitation; CFD; Electrical Submersible Pump; Gas Pocket; Mixture Model; Pump Head; Turbulence Model; Two-Phase Flow; Wall Shear Stress.

Received: January 13, 2021. Revised: July 6, 2021. Accepted: July 14, 2021. Published: August 4, 2021.

\section{Introduction}

Artificial lift mechanism is a process of minimizing Bottom Hole Pressure (BHP) in order to enhance the production rate. Viscous fluid engineers utilize concepts of BHP to produce positive-displacement downhole pump discovered in Progressive Cavity Pump (PCP) as well as downhole centrifugal pump. Electrical Submersible Pump (ESP) is a type of centrifugal pump and the second most utilized artificial lift mechanism; moreover, it is the highest amount in produced volume in two-phase flow. Applications involving Liquefied Natural Gas (LNG) face operational challenges under multiphase flow along with cavitation risk of pump component [1]. Oil and gas industry utilize artificial lift mechanism to boost produced fluid towards the surface during insufficient pressure. Similarly, an alternate artificial lift mechanism, known as gas lift is utilized for weight reduction of hydrostatic column through gas 
insertion in the tubing [2]. Pressure is the primary variable in artificial lift method, and is perpendicular to the surface; whereas, shear stress is parallel to target surface.

Centrifugal pumps transfer centrifugal forces to the working fluids, the fluids losses some of the transferred forces as shear stress between the blade wall and working fluid. Shear stress in continuum mechanics is defined as the stress applied parallel to the cross-section; however, in fluid mechanics, it refers to the effects of viscous flow on surface and between fluids in multiphase flow. Shear stress for fluids in motion is the rate of strain as shown in equation below:

$$
\tau=\mu \frac{\partial \gamma}{\partial t}
$$

where $\tau$ is shear stress, $\mu$ dynamic viscosity, and $\frac{\partial \gamma}{\partial t}$ strain rate.

Once fluid flows in a pipe, velocity of fluid at the fluid-pipe interface is equal to the pipe velocity (which is zero), and is known as no-slip condition. Under open wall flow, the region between boundary and height where the velocity of flow will equal the fluid velocity is known as boundary layer region. In Newtonian and laminar region, shear stress is proportional to strain rate, in which velocity gradient plays a dominant role as per the equation:

$$
\tau(y)=\mu \frac{\partial u}{\partial y}
$$

where $\frac{\partial u}{\partial y}$ is the velocity gradient.

However, study of wall shear stress requires the height at boundary assumed to be zero, so the integration is limited at the surface where the height is zero:

$$
\tau(y=0)=\left.\mu \frac{\partial u}{\partial y}\right|_{y=0}
$$

In Non-Newtonian flow, the viscosity is no longer a constant function with its axis expressed as:

$$
\tau(\vec{u})=\mu(\vec{u}) \nabla \vec{u}
$$

This research investigates the effects of wall shear stress at the impeller blade walls of an ESP; furthermore, it observes the effects of gas bubbles upon the wall shear stress as well as the reduction in area of contact between the wall and the water.

\section{Background Search}

Gonzalez et al. (2002) [3] and Gonzalez \& Santolaria (2006) [4] utilized CFD analysis to simulate the centrifugal pump. A transient model with sliding mesh technique was applied to solve the incompressible Navier-Stokes equation. The results obtained illustrated that the model implemented was able to predict the flow behavior inside the pump. Huang et al. (2010) simulated a three-dimensional single stage centrifugal pump by applying a transient model together with a sliding mesh technique and standard $k-\epsilon$ turbulence model. They investigated the interactions of flow between the impeller (rotor) and the diffuser (stator) inside the pump. The observed results illustrate an interaction pattern between rotor-stator that occurs due to pressure fluctuation and transient flow.

ANSYS Fluent offer various multiphase approaches to solve multiphase problems. The full Eulerian-Eulerian approach, the Volume Of Fluid (VOF) approach and Mixture approach. The difference between these approaches is applied momentum equation. Full Eulerian-Eulerian approach where n-terms of momentum equation are solved based on the number of phases is considered as the most advanced model. Meanwhile the VOF approach uses the fraction of the phases to solve one momentum equation [5]. The mixture model which is the model used in this paper is the summation of all momentum equations [6]. VOF and Mixture models provide accurate results in a time limit much less than the Eulerian-Eulerian model, due to smaller time step and finer mesh requirement for the Eulerian model.

Zhu et al. (2018) [7] used numerical simulation to simulate a three-stage pump in fluent to solve Reynolds Average Navier-Stokes equation (RANS). The Full Eulerian-Eulerian and VOF approaches with $k-\epsilon$ turbulence model were utilized to solve three different cases (case $1 \lambda_{G}=0.35 \%$, case $2 \lambda_{G}=0.56 \%$ and case $3 \lambda_{G}=0.69 \%$ ). The results implemented depict the full Eulerian model was more accurate than the VOF approach in terms of predicting the flow patterns and behavior of bubbles. Figure 1 represents the In-situ contour on second stage impeller for case 2 and case 3 . In case 2 fig. 1-(a) an agglomerated flow pattern was observed due to coalescence of the bubbles at the impeller pressure side; however, increasing the gas fraction further as shown in fig. 2-(b), more coalescence of bubbles predicted which gas pocket was produced. 


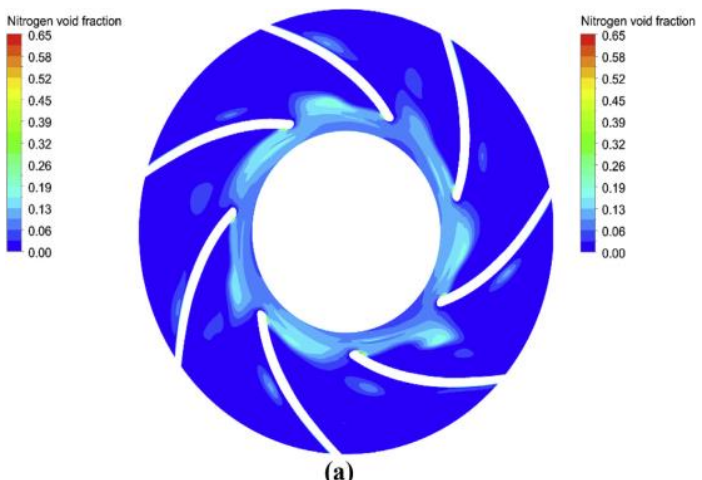

(a)

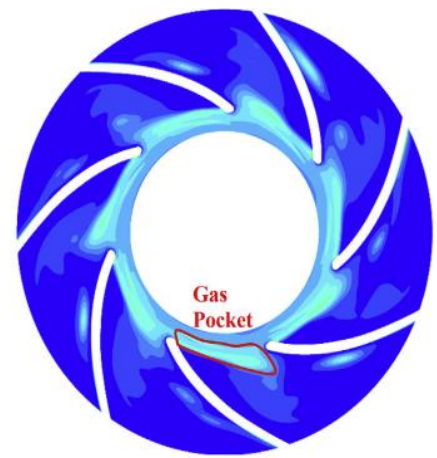

(b)

Fig.1: In-situ contour on second stage impeller, (a) Case $2 \mathrm{t}=7.956 \mathrm{~s}$, (b) Case $3 \mathrm{t}=15 \mathrm{~s}$ [7]

Mizunuma and Nakajima (2007) [8], in the experimental study on shear stress distributions in a centrifugal Blood Pump, surface mounted hot film sensor was utilized to measure the wall shear stress and oil film visualization for shear distribution. From the hot film measurement, in the multiphase flow the shear stress was proportional to rotating speed, while in the boundary layer flow in the oil film method, the casing cover is a spiral flow from outside to inside. Shannak (2008) [9], performed experiments of twophase flow (air-water) on frictional pressure drop of vertical and horizontal as well as smooth and rough pipes, the friction pressure drop was observed to increase with an increase in the relative roughness of the pipe. In the study the parameters considered were the mass flux, the vapor quality, the density and viscosity of each phase, the pipe diameter, pipe orientation and the relative roughness of the pipe. In smooth and rough horizontal and vertical pipes, the frictional pressure drop increases with increasing mass flux, higher vapor quality and higher relative roughness. The influence of relative roughness is more significant especially at higher vapor quality and higher mass flux. Liu et al. (2019) [10] studied friction pressure drop in an inclined pipe at high velocity of two-phase flow (gas-water). The results obtained illustrate the pressure drop increases as the superficial velocities increase, the higher the velocity of the liquid phase the higher the contact with the wall, and the higher the gas flow velocity increases the higher the friction pressure drop. This led to develop a new model of friction pressure drop for different flow patterns.

Zubov et al. (2017) [11], stated that reliability of calculating friction pressure for two-phase flows close to atmospheric pressure in the field of twophase hydrodynamics still remains unsolved. The frictional pressure drop was calculated under low pressure and compared with experimental results. Data from experiment included information on the flow mode boundaries, specific features of the individual structure, the profile of velocity, void fraction, droplet entrainment and deposition, on the distribution of phase interface surface in the flow, on the occurrence and characteristic of various types of instability and on the specific features of two-phase for small diameters. Under low reduced pressures, good results were obtained from using homogeneous model for quasi-homogeneous flow and the annular flow model for high values of void fraction. CFD modeling of flow profiles and interfacial phenomena in two-phase flow in pipes was studied by Ghorai and Nigam (2006) [12]. Two-phase (gas-liquid) flow in pipes was calculated using Eulerian-Eulerian model and commercial CFD package fluent. Parameters such as gas velocity, volume fraction of liquid, and interfacial roughness were considered in the study. Variation from 1.2 to $12.5 \mathrm{~m} / \mathrm{s}$ were done on gas velocity and similarly, from $0.0066 \mathrm{~m} / \mathrm{s}$ to $0.1 \mathrm{~m} / \mathrm{s}$ in liquid. The velocity profile is symmetrical for low superficial gas velocity with a smooth interface; however, at higher velocities ranging from $4.7 \mathrm{~m} / \mathrm{s}$ to $12.5 \mathrm{~m} / \mathrm{s}$ the profile is no longer symmetric and interface became hydro-dynamically rough; moreover, the CFD analysis was validated with experiments on the concept of interfacial roughness for two-phase flow.

\section{Methodology}

\subsection{Geometry}

A replica of GDIWA A Series ESP is modeled as shown in the fig. 2 [13]. The geometry was designed accurately by using CAD program for CFD analysis in Fluent. The curves of impeller blades and diffuser 
outlet are the most effective parts and the most difficult on modeling, table 1 shows some details about impeller blades. The main components of the pump are impeller (rotor), Diffuser (stator), and Volute (fluid path) as show in the fig. 3.

Table 1: details of the impeller blades

\begin{tabular}{|l|c|c|}
\hline Parameter & Symbol & Value \\
\hline Blade height $(\mathrm{mm})$ & $\mathrm{H}$ & 11 \\
\hline Number of blades & $\mathrm{n}$ & 4 \\
\hline Impeller Inlet angle $\left(^{\circ}\right)$ & $\beta_{1}$ & 97 \\
\hline Impeller outlet angle $\left(^{\circ}\right)$ & $\beta_{2}$ & 44 \\
\hline
\end{tabular}

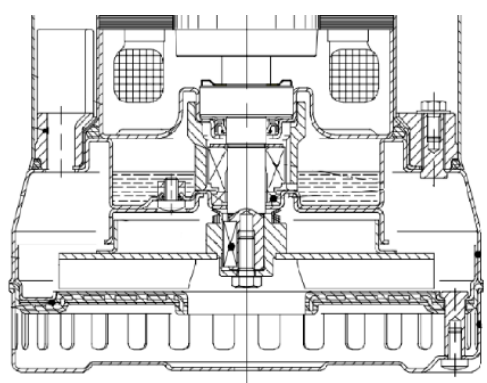

Fig.2: Sectioning of GDIWA A Series ESP pump [13]

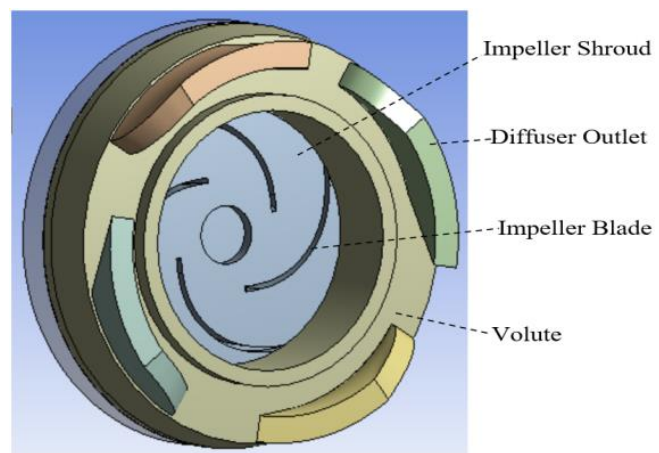

Fig.3: Assembled pump geometry for CFD analysis

\subsection{Mesh Independency Test}

Mesh independency test was performed to optimize the best mesh number that provides accurate results in a limited time frame. The performance curve of the pump was provided by the manufacture at 2850 rpm rotation speed [14]; moreover, $150 \mathrm{~L} / \mathrm{min}$ flow rate and 2850 tetrahedron mesh type as shown in fig. 4 were selected as operating point for single-phase flow while the number of elements is varied by 1.5 , 3, 5, 7 and 9 million elements. The results show that at 3 million elements the percentage of error dropped by $3 \%$ from 1.5 million elements while the simulating time is increased by 5 minutes; meanwhile, as the number of elements increased further the simulation time will increase and the reduction percentage is unfeasible due to trade-off with reduction in error. Fig. 5 shows the mesh independency test conducted for this research paper.

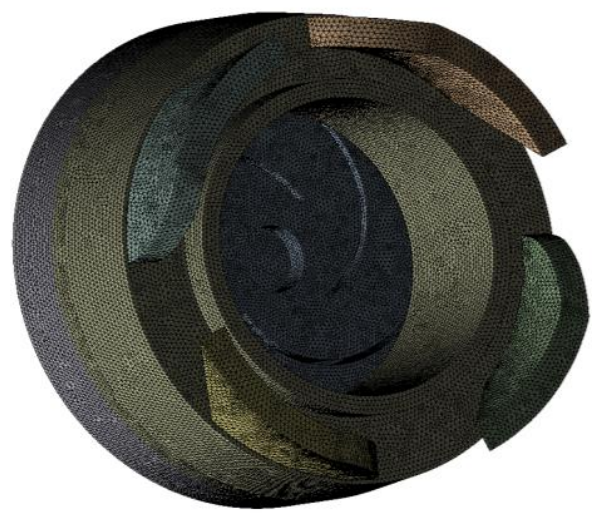

Fig.4: Tetrahedron Mesh Type Pressure Head vs Mesh Elements

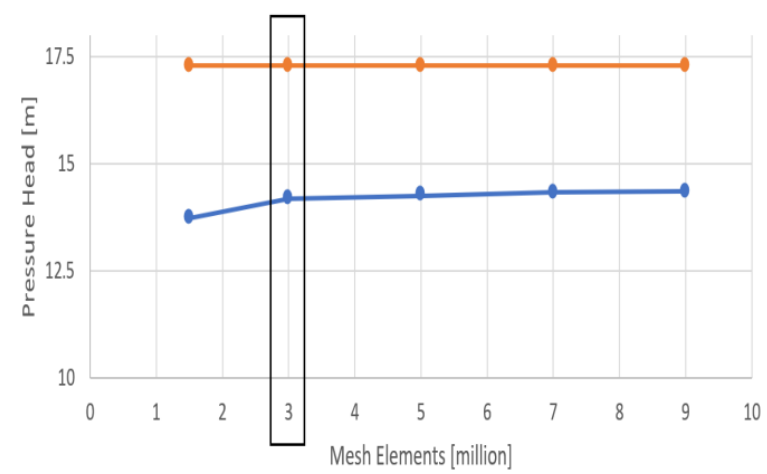

Fig.5: Mesh Independency Test

\subsection{Setting up the Two-Phase flow Model}

Two-Phase flow models are generally more complicated compared to single-phase flow model in defining parameters, since more boundary conditions are required to be defined in fluent; moreover, fluent offers various models for solving Navier-Stokes equation based on the study focused on. Table 2 represent more details of the models used and the boundary conditions specified for each component.

Table 2. Details of the CFD analysis model

\begin{tabular}{|l|l|}
\hline $\begin{array}{l}\text { Model } \\
\text { Turbulence } \\
\text { model }\end{array}$ & Method \\
\hline $\begin{array}{l}\text { Multiphase } \\
\text { model }\end{array}$ & Mixture model (implicit) \\
\hline $\begin{array}{l}\text { Body forces } \\
\text { formulation }\end{array}$ & Implicit body forces \\
\hline Interface & Sharp/Dispersed \\
\hline
\end{tabular}




\begin{tabular}{|c|c|}
\hline modeling & \\
\hline Water, Air & $\begin{array}{l}\text { Primary phase, Secondary } \\
\text { phase }\end{array}$ \\
\hline $\begin{array}{l}\text { Boundary } \\
\text { Conditions }\end{array}$ & Value \\
\hline Velocity inlet & $\begin{array}{l}0.20938 \mathrm{~m} / \mathrm{s} \text { equivalent to } 20 \\
\mathrm{~L} / \mathrm{min}\end{array}$ \\
\hline Outlet boundary & $\begin{array}{l}\text { Pressure outlet (gauge } \\
\text { pressure) }\end{array}$ \\
\hline $\begin{array}{l}\text { Rotational speed } \\
\text { (impeller) }\end{array}$ & $\begin{array}{l}500,900,1500,2000,2500 \\
\mathrm{rpm}\end{array}$ \\
\hline Solids boundary & $\begin{array}{l}\text { Stationary relative to adjacent, } \\
\text { no-slip condition }\end{array}$ \\
\hline $\begin{array}{l}\text { Water density } \\
\text { and fraction }\end{array}$ & $998 \mathrm{~kg} / \mathrm{m}^{3}, 99 \%$ \\
\hline $\begin{array}{l}\text { Air density and } \\
\text { fraction }\end{array}$ & $1.225 \mathrm{~kg} / \mathrm{m}^{3}, 1 \%$ \\
\hline Gravity & $\mathrm{Y}$ direction $\mathrm{g}=-9.81 \mathrm{~m} / \mathrm{s}^{2}$ \\
\hline Time step size & $0.0001 \mathrm{~s}$ \\
\hline
\end{tabular}

This model consists of turbulence flow; therefore, it is crucial to focus the effects of turbulence on the wall. $K-\omega$ shear stress transport (SST) is a two equations eddy viscosity turbulence model that provides accurate results on adverse pressure gradient and near wall effects [15]. The standard $K-\omega$ equation is shown as the following:

$$
\begin{aligned}
& \frac{\partial k}{\partial t}+U_{j} \frac{\partial k}{\partial x_{j}}=P_{k}-\beta^{*} k \omega \\
&+\frac{\partial}{\partial x_{j}}\left[\left(v+\sigma_{k} v_{T}\right) \frac{\partial k}{\partial x_{j}}\right]
\end{aligned}
$$

where $v_{T}$ is the kinematic eddy viscosity, and the transport equations of the $K-\omega \mathrm{SST}$ are [16]:

$$
\begin{aligned}
\frac{\partial}{\partial t}(\rho k)+\frac{\partial}{\partial x_{i}}\left(\rho k u_{i}\right)+Y_{k}-S_{k} \\
=\frac{\partial}{\partial x_{j}}\left[\left(\mu+\frac{\mu_{t}}{\sigma_{k}}\right) \frac{\partial k}{\partial x_{j}}\right]+\tilde{G}_{k}
\end{aligned}
$$

$$
\begin{aligned}
\frac{\partial}{\partial t}(\rho \omega)+\frac{\partial}{\partial x_{i}}( & \left(\rho u_{i}\right) \\
& =\frac{\partial}{\partial x_{j}}\left[\left(\mu+\frac{\mu_{t}}{\sigma_{\omega}}\right) \frac{\partial \omega}{\partial x_{j}}\right] \\
& +G_{\omega}-Y_{\omega}+D_{\omega}+S_{\omega}
\end{aligned}
$$

where

$$
\begin{array}{l|l}
\tilde{G}_{k} & \begin{array}{l}
\text { Generation of turbulence kinetic } \\
\text { energy due to mean velocity } \\
\text { gradient }
\end{array}
\end{array}
$$

$$
\begin{array}{r|l}
G_{\omega} & \text { Generation of } \omega \\
Y_{k} \& Y_{\omega} & \text { Dissipation of } k \text { and } \omega \\
D_{\omega} & \text { Cross-diffusion term } \\
S_{k} \& S_{\omega} & \text { User defend source terms }
\end{array}
$$

The multiphase models are varied in the continuity equation, for the Mixture model the continuity equation form as:

$$
\frac{\partial}{\partial t} \sum_{k=1}^{n}\left(\alpha_{k} \rho_{k}\right)+\nabla \cdot \sum_{k=1}^{n}\left(\alpha_{k} \rho_{k} u_{k}\right)=0
$$

where $\alpha$ is the fraction and the subscript $\mathrm{k}$ is the phase. The general Navier-Stokes Equation can be written as the following:

$\frac{d}{d t} \int_{V} \rho \phi d V \int_{\partial V} \rho \phi\left(\vec{u}-\overrightarrow{u_{g}}\right) \cdot d \vec{A}=$

$\int_{\partial V} \Gamma \nabla \phi \cdot d \vec{A}+\int_{V} S_{\phi} d V$

\section{Results and Discussion}

Figure 6 represents the results of air volume fraction at mid span of impeller blades, $20 \mathrm{~L} / \mathrm{min}$ water flow rate and $1 \%$ air volume fraction. At low rotational speed of $500 \mathrm{rpm}$ in fig. 6-(a) a large gas pocket is concentrated at the impeller inlet which choke the flow inside the pump with bubbles accumulating the blades pressure side to produce a gas pocket [17]. This leads to reduction in kinetic energy utilized to breakdown the bubbles concentration. As the rotational speed increases further as illustrated in fig. 6-(b), the large pocket begins to collapse to smaller bubbles with limited volume fraction at the blades pressure side. For higher rotation speeds $1500 \mathrm{rpm}$ and $2000 \mathrm{rpm}$ the gas pockets collapsed to small bubbles due to decrease in bubbles size as shown in fig. 6-(c) and (d); moreover, the bubbles moved towards the volute with higher fraction of $2 \%$. Fig. 6(e) illustrate the air volume fraction contour at maximum rotation speed $2500 \mathrm{rpm}$. It is clearly visible that large gas pocket and the concentration of bubbles at blades are collapsed totally due to high kinetic energy produced from the rotated impeller, and the high drag force applied by the liquid phase on the gas phase [18]. 


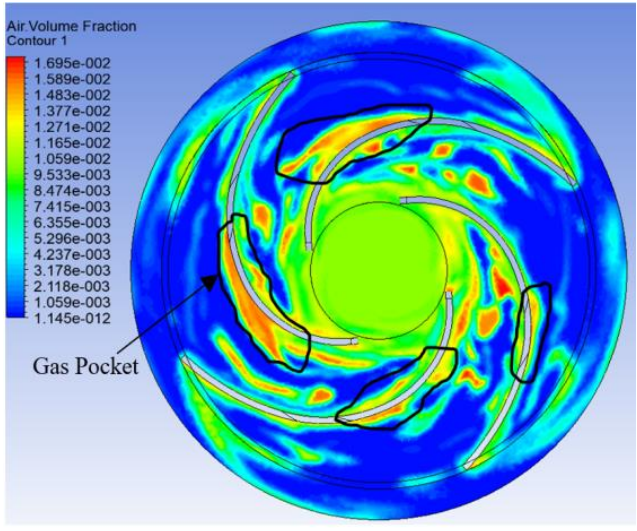

(a) $500 \mathrm{rpm}$

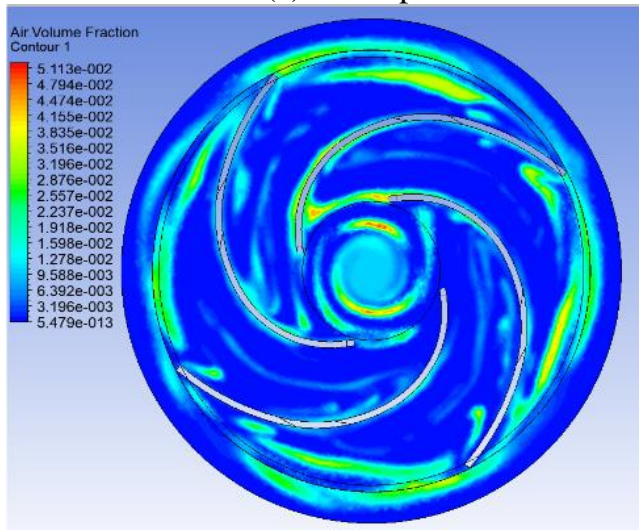

(c) $1500 \mathrm{rpm}$

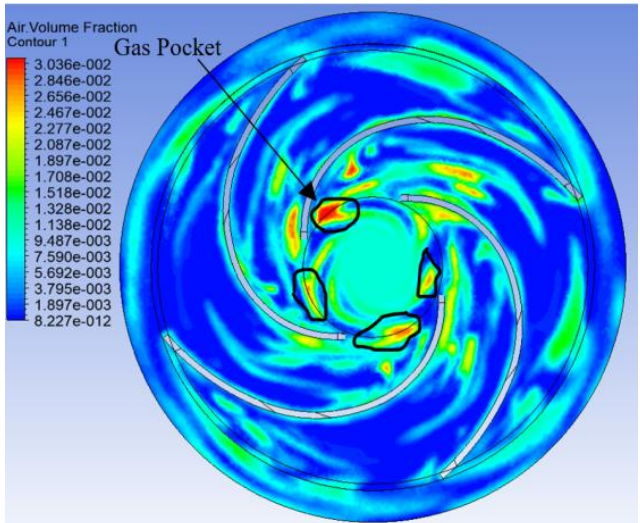

(b) $900 \mathrm{rpm}$

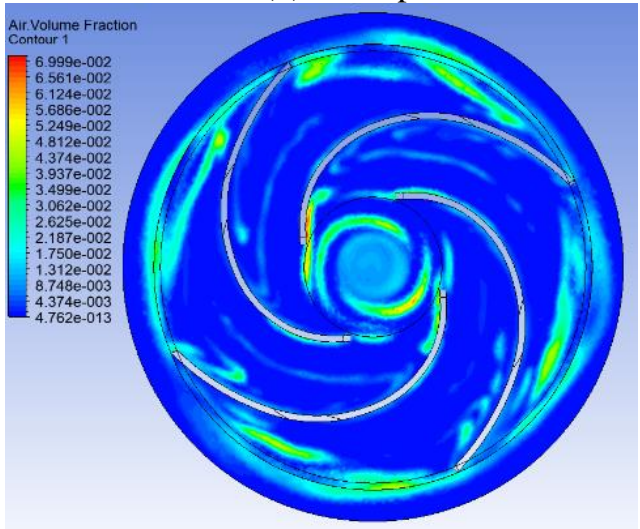

(d) $2000 \mathrm{rpm}$

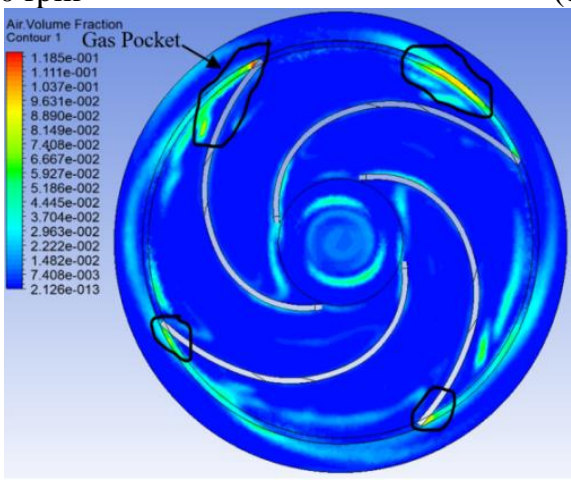

(e) $2500 \mathrm{rpm}$

Fig.6: Air Volume Fraction Contours at mid span impeller blades

The results in this section focus on the effects of wall shear stress (WSS). WSS behavior on the pump performance plays a dominant role, as the rotation speed increases the friction pressure also increase [10]. Fig. 7 illustrates the Wall Shear Stress comparison of single-phase flow results at the impeller blade. The light blue color represents the 500 -rpm rotation speed, pink is $900 \mathrm{rpm}$, black is $1500 \mathrm{rpm}$, red is $2000 \mathrm{rpm}$ and dark blue is 2500 $\mathrm{rpm}$. It can be observed that WSS is proportional to the rotation speed due to increases on the velocity gradient as per the equation below:

$$
\tau=\mu \frac{\partial u}{\partial y}
$$

The velocity gradient at the impeller blade increases as the length of the blade increases, which in turn results in the increase of wall shear stress. The values of WSS are fluctuating; however, the overall trend of the graph depicts an increase in WSS. 


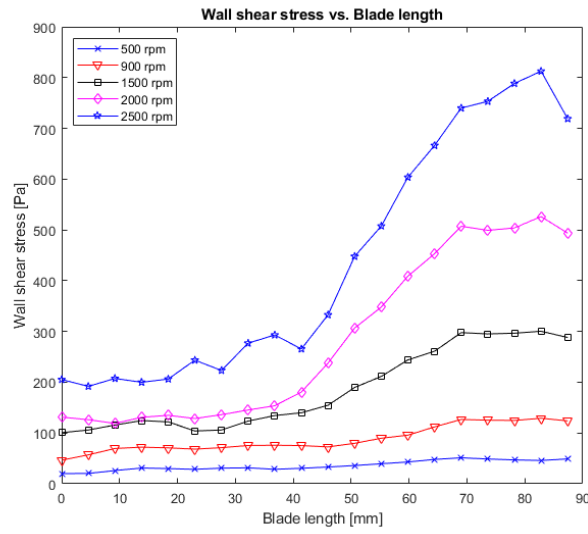

Fig.7: Wall Shear Stress of single-phase flow at different rpm's vs blade length

Two-phase flow wall shear stress has the same trend that of the single-phase at different rotational speed. Figure 8 below depicts the two-phase flow results of WSS vs the blade length. At different rotational speed from the plot of WSS vs blade length when the blade length beyond $85 \mathrm{~mm}$ the wall shear stress drops rapidly due to the high velocity at tips as well as the flow separation and recirculation occurring at the blade tips [19]. Figure 9 shows the velocity vector at mid span of impeller blade of the $1500 \mathrm{rpm}$ rotation speed and two-phase flow. The zoom in figure show that the velocity at the blade tip always recirculates due to the high velocity of the blade as well as the generated wake region [20].

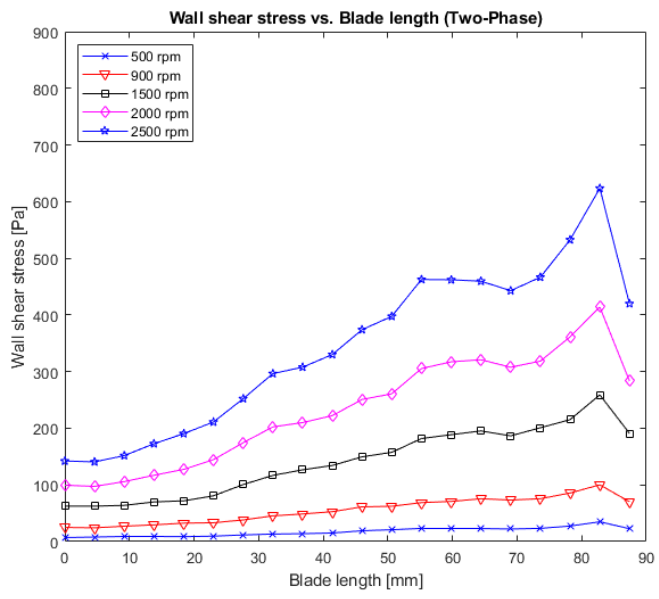

Fig.8: Wall Shear Stress of two-phase flow at different rpm's vs blade length

Figure 10 illustrate the association between single and two-phase flow wall shear stresses at $1500 \mathrm{rpm}$. The blue color denotes the single-phase flow and red for the two-phase flow. Both trends increase the WSS values as the blade length increases; however, singlephase flow values are higher than that of two-phase flow, which is due to bubble's concentration at the impeller blade plus the viscosity effects between both flows [21]. The two-phase flow results show that air bubbles take place around the impeller blades, when the impeller rotates the bubble restrict the area of contact between the liquid (water) and the wall, which leads to reduction of wall shear stress. The detailed over view of this is illustrated in fig. 11, where a high concentration of air fraction around the blade root is more than the blade tip. Furthermore, in two-phase flow the viscosity is no more constant due to mixture viscosity [22]. 


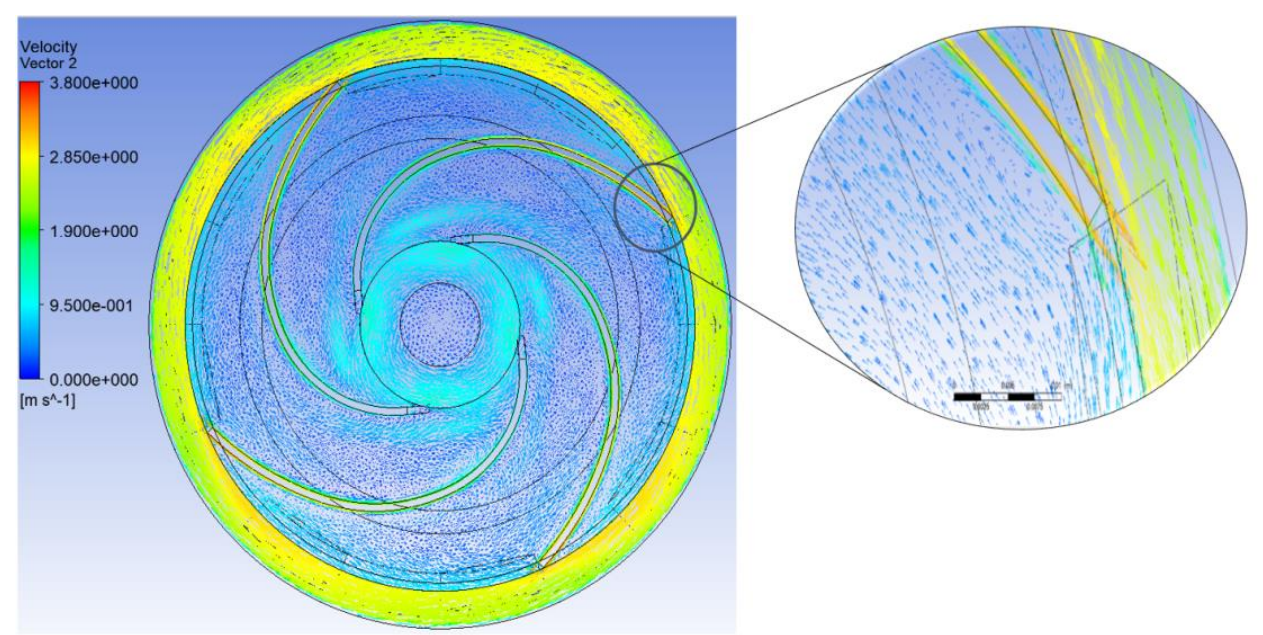

Fig.9: Velocity vector at mid span of the blade, $1500 \mathrm{rpm}$ and two-phase flow

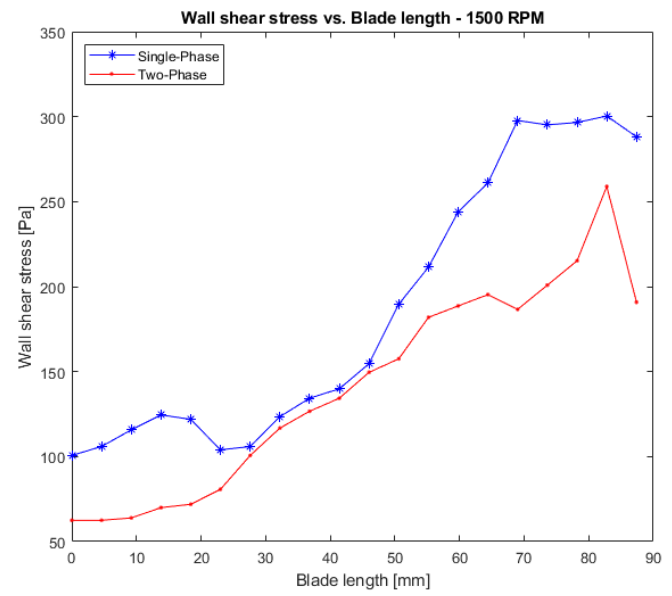

Fig.10: Wall Shear Stress for $1500 \mathrm{rpm}$ at different flow vs blade length

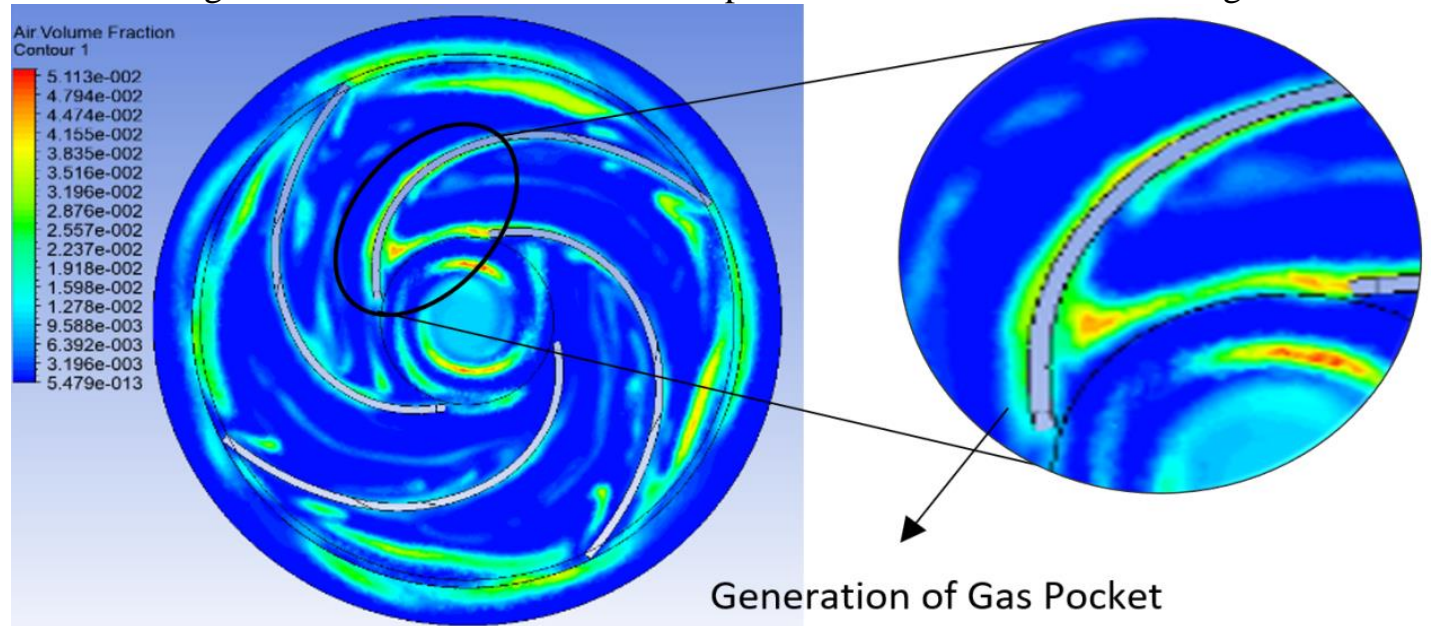

Fig.11: Detailed over view of bubble formation at $1500 \mathrm{rpm}$

\section{Conclusion}

The model simulated in this research was able to predict wall shear stress effects on the impeller blades for single-phase and two-phase flow. The $K-\omega$ SST Turbulence model and the mixture approach multiphase model with the sliding mesh technique 
were utilized to visualize the flow patterns inside the pump. The rotation speed varied by 500, 900, 1500, 2000 and $2500 \mathrm{rpm}$ while the water flow rate and gas fraction were kept constant with $20 \mathrm{~L} / \mathrm{min}$ and $1 \%$, respectively in all simulation. Finally, the results depict that the single-phase flow wall shear stress values are always higher than two-phase flow in all rotational speeds.

\section{Acknowledgement:}

We would like to thank Abu Dhabi Award for Research Excellence under ASPIRE/Advanced Technology Research Council for the research funding.

\section{References:}

[1] S. Shahid, S. S. Dol, A. Hasan, O. Kassem, M. Gadala and M. Aris, "A Review of Electrical Submersible Pump Head Losses and Methods to Analyze Two-Phase Performance Curve," WSEAS Transaction on Fluids Mechanics, vol. 16, pp. 14-31, 2020.

[2] K. Dou, Q. Liu and L. Meng, "Study of Fluid Flow, Heat Transfer and Surface Wave Vibration Behaviour for Steel in Continuous Casting Process using Mathematical Modelling and Physical Modelling," WSEAS Transaction on Heat and Mass Transfer, vol. 14, pp. 153157, 2019.

[3] J. Gonzalez, J. Fernandez, E. Blanco and C. Santolaria, "Numerical Simulation of Dynamic Effects Due to Impeller-Volute Interaction in a Centrifugal Pump," Journal Fluid Engineering, vol. 124, pp. 348-355, 2002.

[4] J. Gonzalez and C. Santolaria, "Unsteady Flow Structure and Global Variables in a Centrifugal Pump," Journal Fluid Engineering, vol. 128, pp. 937-946, 2006.

[5] T. Watanabe, "Numerical Simulation of Droplet Combusion Using Volume-of-Fluid Method," WSEAS Transaction on Heat and Mass Transfer, vol. 14, pp. 38-44, 2019.

[6] A. Fluent, ANSYS Fluent Theory Guide 18.0, USA: Ansys. lnc, 2017.

[7] J. Zhu, H. Zhu, J. Zhang and H.-Q. Zhang, "A Numerical Study on Flow Patterns Inside an
Electrical Submersible Pump (ESP) and Comparison with Visualization Experiments," Journal of Petrolum Science and Engineering , vol. 173, pp. 339-350, 2018.

[8] H. Mizunuma and R. Nakajima, "Experimental Study on Shear Stress Distributions in a Centrifugal Blood Pump," International Center for Artificial Organs and Transplantation, vol. 31, pp. 550-559, 2007.

[9] B. A. Shannak, "Frictional Pressure Drop of Gas liquid Two-Phase Flow in Pipes," Nuclear Engineering and Design, vol. 238, pp. 32773284, 2008.

[10] Z. Liu, R. Liao, W. Luo, J. X. F. Ribeiro and Y. $\mathrm{Su}$, "Friction Pressure Drop Model of GasLiquid Two-Phase in an Inclined Pipe with High Gas and Liquid Velocities," AIP Advances 9, vol. 085025, pp. 1-10, 2017.

[11] N. O. Zubov, O. N. Kov, V. V. Yagov and L. A. Sukomel, "Prediction of Friction Pressure Drop for Low Pressure Two-Phase Flows on the Basis of Approximate Analytical Models," Thermal Engineering, vol. 64, no. 12, pp. 898-911, 2017.

[12] S. Ghorai and K. Nigam, "CFD Modeling of Flow Profiles and Interfacial Phenomena in Two-Phase Flow in Pipes," Chimecal Engineering and Processing, vol. 45, pp. 55-65, 2005.

[13] S. Shahid, A. Q. Hasan, S. S. Dol, M. S. Gadala and M. S. Aris, "Effects of Near-Wall Vorticies on Wall Shear Stress in a Centrifugal Pump Impeller," WSEAS Transaction on Fluid Mechanics, vol. 16, pp. 37-47, 2021.

[14] O. M. Kassem, A. Q. Abdullah, S. S. Dol, M. S. Gadala and M. S. Aris, "CFD Analysis of Electrical Submersible Pump Handling SinglePhase Flow," Platform: A Journal of Engineering, vol. 4, no. 4, pp. 26-34, 2020.

[15] H. B. Chan, T. H. Yong, P. Kumar, S. K. Wee and S. S. Dol, "The Numerical Investigation on the Effects of Aspect Ratio and Cross-Sectional Shaper on the Wake Structure Behind a Cantilever," ARPN Journal of Engineering and Applied Science, vol. 11, no. 16, pp. 9922-9932, 2016. 
[16] S. S. Dol, S. K. Wee, H. B. Chan and P. Kumar, "The Effects of Flexible Vortex Generators on the Wake Structures for Improving Turbulence," in IOP Conference Series: Materials Science and Engineering, vol. 715, No, 012070, 2020.

[17] O. Shudryk, N. Shevchenko and O. Koval, "Investigation of Gas-Liquid Mixture Flow in the Stage Submersible Pump," WSEAS Transaction on Heat and Mass Transfer, vol. 14, pp. 107-112, 2019.

[18] S. F. Wong and S. S. Dol, "Turbulence Charactristics Study of the Emulsified Flow," WSEAS Transaction on Heat and Mass Transfer, vol. 14, pp. 45-50, 2019.

[19] T. Halawa, M. Alqaradawi, O. Badr and M. Gadala, "Numerical Investigation of Rotating Stall Charactristics and Active Stall Control in Centrifugal Comperessor," in ASME 2014 Power Conference, Maryland USA, 28-31 Jul. 2014.

[20] S. S. Dol, S. K. Wee, C. H. Bin and P. Kumar, "Turbulence Characteristics behind a Flexible Vortex Generator," WSEAS Transaction on Fluid Mechanics, vol. 14, pp. 1-7, 2019.

[21] J. Zhu and H.-Q. Zhang, "Mechanistic Modeling and Numerical Simulation of In-situ Gas Void Fraction inside ESP Impeller," Journal of Natural Gas Science \& Engineering, vol. 36, pp. 144-154, 2016.

[22] M. Y. Sikkandar, N. M. Sudharsan, S. S. Begum and E. Y. K. NG, "Comutational Fluid Dynamic:
A Technique to Solve Complex Biomedical Engineering Problems - A Review," WSEAS Trensaction on Biology and Biomedicine, vol. 16, pp. 121-137, 2019.

\section{Contribution of Individual Authors to the Creation of a Scientific Article (Ghostwriting Policy)}

- Abdulqader Hasan and Salman carried out Conceptualization, data curation, validation, and writing origin draft.

- Mohammed Alavi carried out methodology and writing origin draft.

- Dr. Sharul Sham Dol, Dr. Mohamed S. Gadala and Dr. Mohd Shiraz Aris carried out project supervision and project administration.

\section{Sources of Funding for Research Presented in} a Scientific Article or Scientific Article itself

We would like to thank Abu Dhabi Award for Research Excellence under ASPIRE/Advanced Technology Research Council for the research funding.

\section{Creative Commons Attribution License 4.0 (Attribution 4.0 International, CC BY 4.0)}

This article is published under the terms of the Creative Commons Attribution License 4.0 https://creativecommons.org/licenses/by/4.0/deed.en_ US 